\title{
Evaluating dyspnoea in chronic obstructive pulmonary disease
}

\section{Kian-Chung Ong}

Chestmed Pte Ltd, Mount Elizabeth Medical Centre, Singapore

Received: December 22, 2020

Revised: May 18, 2021

Accepted: May 18, 2021

Corresponding author:

Kian-Chung Ong

Chestmed Pte Ltd, Mount

Elizabeth Medical Centre,

3 Mount Elizabeth, 228510,

Singapore

Tel: +65-6235-1466

E-mail: ongkc@chestmed.com.sg

\begin{abstract}
Dyspnoea is the most common and troubling symptom in patients with chronic obstructive pulmonary disease (COPD) and the severity of the symptom engenders negative impacts on health status, exacerbation rate, healthcare resource utilization and prognosis. Therefore, alleviating dyspnoea is a primary treatment goal for most patients with stable COPD. The objective of improving dyspnoea is supreme in the management of COPD as few therapeutic interventions are disease-modifying in curtailing the underlying problem of accelerated loss of lung function leading to premature disability or death. However, in spite of the well-established fundamental mechanisms that induce dyspnoea in healthy individuals and patients with COPD, as well as the proven effectiveness of multiple and varied therapeutic interventions in reducing dyspnoea in such patients, the clinical evaluation and quantification of dyspnoea remain imprecise. Subjective measurement tools currently used in clinical practice are incapable of grading both immediate and longer-term changes in dyspnoea simultaneously, and repetitive scoring using questionnaires is burdensome for both the patient and observer. This paper reviews the current understanding and management of dyspnoea in COPD and discusses potential improvements in evaluating dyspnoea with the aim of achieving personalization of care for patients with COPD.
\end{abstract}

Keywords: Exercise test; Health status; Lung; Spirometry; Surveys and questionnaires
This is an Open Access article distributed under the terms of the Creative Commons Attribution Non-Commercial License (https:// creativecommons.org/licenses/ by-nc/4.0/).

\section{INTRODUCTION}

Chronic Obstructive Pulmonary Disease (COPD) is a major cause of morbidity and mortality [1]. It is currently the third leading cause of death worldwide, and even for this year, historically different in many ways due to the coronavirus disease 2019 (COVID-19) pandemic, COPD persists in claiming more lives than respiratory tract infections. COPD is also a leading cause of morbidity worldwide, and imposes huge economic and social burden in many countries.

Global evidence-based management guidelines for COPD endorse the treatment of dyspnoea as a primary goal [1]. This is appropriate and necessary since dyspnoea, together with associated physical limitation, are by far the most troubling symptoms, according to patients with COPD [2]. More than $80 \%$ of stable COPD patients complain of dyspnoea [3]. The current Global Initiative for Chronic Obstructive Lung Disease (GOLD) recommendations for initial 
pharmacotherapy for stable COPD are targeted at the two treatable traits of symptoms and exacerbation rates. Since most COPD patients do not suffer from repeated bouts of exacerbation [4], pharmacotherapy for most COPD patients is presently based on their symptoms. GOLD recommends that these symptoms be simply evaluated in clinical practice using either the modified Medical Research Council (mMRC) dyspnoea scale [5] or the COPD Assessment Test (CAT) [6], the latter including measurement of symptoms other than dyspnoea, such as cough and sleep disturbance. Patients with mMRC dypsnoea score of more than 2 or CAT score greater than 10 are considered more symptomatic and requiring more effective medication. These 'cut-off' values chosen for categorizing patients using these symptom scoring tools are completely arbitrary. After the initial pharmacotherapy is prescribed and during follow-up review, the initial treatment is to be adjusted-if symptoms persist despite initial treatment, the clinician is advised to review the prescribed medication, consider non-adherence to prescribed treatment and explore non-pharmacological treatment modalities such as pulmonary rehabilitation (PR).

One may surmise that, based on current management guidelines, most COPD patients are treated predominantly according to their symptoms. This may be unavoidable since most of the widely available therapeutic options are not conclusively proven to be disease-modifying (other than smoking cessation) and there are no established biomarkers to guide therapy in stable COPD patients, who do not suffer from frequent episodes of exacerbation. In fact, lung function testing, such as spirometry that is useful in diagnosis and grading severity of airway obstruction, is not well correlated with patient's symptoms [7], health status [8,9], or treatment response [10]. This begs the question of how reliable are the commonly used symptom-scoring tools (in particular those for measurement of dyspnoea) in evaluating patients' dyspnoeic sensation?

\section{PATHOPHYSIOLOGICAL MODEL OF DYSPNOEA IN COPD}

Before one considers how dyspnoea may be quantified, it is helpful to first review the pathophysiological processes in COPD that induce dyspnoea in patients. According to the most widely accepted hypothesis [11], dyspnoeic sensation is considered to arise whenever increasing ventilatory demand causes a neuro-mechanical dissociation in the respiratory system leading to efferent-afferent mismatching. In healthy individuals at rest, respiratory centres in the cerebral cortex and brainstem function as controllers of breathing by producing neural signals that are transmitted to the muscles of ventilation, viz., the diaphragm. The sensory afferents include direct chemo-stimulation of medullary centres by oxygen desaturation, increased carbon dioxide production and acidosis, as well as proprioceptive receptors in respiratory and locomotor muscles.

Conscious awareness of the level of neural respiratory drive (NRD) plays a very important role in the perception of breathlessness -the brain 'expects' a certain pattern of ventilation and feedback for a given level of NRD. Deviation of the afferent signal from what is expected causes or intensifies the sensation of breathlessness [11]. The degree of the sensation is correlated with the drive to breathe. Beyond a certain threshold, escalating breathing difficulty is experienced as an imminent threat to life or well-being, demanding immediate behavioural action [12]. Assuming that the efferent-afferent loop remains intact regulating breathing in COPD patients, the difference in the mechanism producing dyspnoea in COPD arise mainly from the disordered pulmonary mechanics in such patients.

\section{Impact of COPD on pulmonary mechanics}

The underlying pathophysiologic mechanism in COPD is increased airflow limitation during expiration. Increased airway resistance contributed by inflammatory changes in the large and small ( $<2 \mathrm{~mm}$ in diameter) airways lead to air trapping during expiration [11]. The loss of elastic recoil due to emphysema and parenchymal destruction causes static hyperinflation as the reduced inward lung recoil pressure requires a greater volume to balance the (outward) chest wall recoil, and, therefore, functional residual capacity increases. At rest, static hyperinflation leads to change in pulmonary mechanics-lower tidal volumes and lower lung compliance. Hyperinflation also weakens the inspiratory muscles to contract and generate negative intrathoracic pressure $[11,13]$.

During exercise, the above changes in lung mechanics are worsened. Further air-trapping results from increased respiratory rate and reduced expiratory time. With increased air trapping, the operating lung volumes (ratio of tidal volume to inspiratory capacity) are reduced, so much so that the only way to increase minute ventilation during exercise is to increase respiratory rate, further adding to air trapping and hyperinflation. This dynamic hyperinflation also causes increased work of breathing in two ways: first, the end-expiratory lung volume (EELV) rises above the relaxation volume of 
the chest wall, so that at EELV the chest wall will recoil inwards, increasing in the inspiratory elastic load; second, increased intrinsic positive end-expiratory pressure, developing as a consequence of air trapping requires an additional inspiratory effort before intrathoracic pressure falls below atmospheric pressure and inspiratory airflow can begin [11, 13].

\section{Increased respiratory drive in COPD}

Not only is the ventilatory capacity of COPD patients reduced, the ventilatory demand in these patients is higher than in healthy individuals at rest and at the same intensity of exercise. The higher ventilatory demand is the consequence of inefficiency of ventilation resulting from ventilation-perfusion mismatch and increased physiological dead space in the lungs resulting from pathological changes in the lungs. Furthermore, COPD patients have higher metabolic demands contributed by higher lactic acid production due to physical deconditioning or poor oxygen delivery to exercising skeletal muscles because of concomitant cardiovascular disease. The inspiratory neural drive (IND), which is measured by electromyography (EMG) of the diaphragm provides the strongest correlation with dyspnoeic sensation in human studies [13]. In addition to ventilatory capacity and metabolic demand, NRD is also affected by other factors such as emotion, stimulation of upper airway receptors, and pulmonary stretch receptors [11]. As in healthy subjects, it is the neuromechanical uncoupling of increasing neural active drive from proportionate mechanical responses of respiratory muscles that occur with physical activity, leading to efferent-afferent mismatching, that drives exertional dyspnoea in COPD.

\section{The perception of dyspnoea in COPD}

The above brief description of pathophysiological processes may help elucidate the common symptom of exertional dyspnoea in COPD patients but not all of it. The perception of dyspnoea may also be also altered in patients with COPD. The American Thoracic Society statement on dyspnoea defined it as consisting of "qualitatively distinct sensations that vary in intensity" [14]. There are at least four different somatic descriptors of breathlessness among COPD patients: (1) perceived sense of increased work or effort; (2) sense of chest tightness; (3) air hunger or an uncomfortable urge to breathe; and (4) unsatisfied inspiration [15]. Although these descriptors may not be unique to COPD and may be shared by patients with other diseases, these various sensations can be accounted for by the pathophysiological processes detailed above and what patients tell their doctors about what makes them feel breathless may suggest to clinicians the likely contributory factors and suitable remedies in cases of new-onset dyspnoea.

Psychological factors may arise consequent to the pathophysiological processes in COPD and there may exist an interplay between these psychological factors and the perception of dyspnoea in COPD. There is evidence that, although interrelated, intensity (sensory) and emotional (affective) components of breathlessness are processed separately by different parts of the brain, and healthy subjects are able differentiate between sensory (intensity) and affective (unpleasantness) components of breathlessness during exercise [11]. Likewise, it has been shown that COPD patients are able to distinguish between breathlessness-related anxiety and increase in intensity of breathlessness during exercise [16].

\section{THE NEED FOR PRECISE MEASUREMENT TOOL OF DYSPNOEA}

A greater appreciation of the underlying pathophysiological mechanisms of dyspnoea in COPD may account for the propinquity of the pulmonologists' perception of disease impact to patients' own, as compared to observations made by general practitioners [17]. Dyspnoea is a symptom that is complex and multidimensional and the evaluation and quantification of dyspnoea in COPD remains challenging, and requires more than what patients can express to their doctors or what their doctor can obtain from their patients.

Although cognizant of the fact that assessment and quantification of any subjective experience such as dyspnoea is difficult, clinicians are reliant on patient-reports in order to quantify the intensity and impact of dyspnoea. The utility and limitations of such standardized measurement tools of dyspnoea have been discussed [18]. In general, there are three distinct sets of these measurement tools: (1) short-term measures of intensity of dyspnoea, e.g., Borg scale and visual analog scale; (2) situational measures, e.g., the baseline dyspnoea index, the transition dyspnoea index (BDI-TDI) and the mMRC dyspnoea scale; (3) impact measures of dyspnoea, e.g., the Chronic Respiratory Questionnaire. The first set deals with current states of breathlessness and are useful for evaluating treatments that have rapid effects on dyspnoea. Thus, they are excellent for documenting dyspnoea with exercise and response to short-term interventions in the laboratory setting. The second group of measures quantify dys- 
pnoea in response to specified standard set of activities such as walking on level ground or incline, or during daily efforts and tasks. They are useful for measuring changes in dyspnoea with longer-term interventions such as PR and long-acting bronchodilators. Currently, the most widely used instruments for assessing the impact of dyspnoea are the BDI-TDI and the Medical Research Council (MRC) Questionnaire [19]. However, situational tools are not able to separate activity level from dyspnoea intensity since the activities are standardized for comparison purposes [18]. Also, the MRC Questionnaire, though simple for scoring, is limited by its scaling (five-points instead of a minimum of seven for precision). Another limitation among all these patient-reported measurement tools is that only one known tool (breathing descriptor tool) can evaluate the individual qualities of the dyspnoea sensation in the American Thoracic Society definition of dyspnoea, but even so, its use is limited as factors such as ethnicity may produce differences in qualities of dyspnoea sensations reported [18]. The third set of measures do not quantify dyspnoea per se but rather of its impact, e.g., on health status and functional status. Thus, they generally are even less precise tools of dyspnoea evaluation.

The limitation of current measurement tools is pointedly expressed in a recent review article on this topic: "The symptom of dyspnoea is a uniquely personal experience and difficult to quantify numerically with magnitude-of-tasks questionnaires (mMRC) or even multicomponent questionnaires which evaluate intensity, quality, affective dimension, and impact on quality of life (BDI-TDI)" [13].

\section{PRESENT AND FUTURE CONSIDERATIONS}

Notwithstanding the complexity involved in patients' perception of dyspnoea and the difficulty in expression of the sensation to observers, it is imperative that clinicians be able to accurately evaluate and quantify dyspnoea for the advancement in COPD management. Relieving dyspnoea remains a primary treatment goal of COPD not just because it is the most vexing symptom for most patients [2] but also because dyspnoea portends a higher risk of exacerbation [20] and poorer prognosis. In fact, dyspnoea is a more accurate predictor of mortality in COPD compared with the degree of airway obstruction measured by spirometry, the standard diagnostic tool for COPD [21].

Inhaled long-acting bronchodilators provide the cornerstone treatment for most COPD patients with persistent symptoms, as outlined above. Great strides have been made in recent years in providing evidence of the effectiveness of dual-bronchodilator therapy over monotherapy in significantly improving lung function, dyspnoea scores and health status [22]. However, improvements in symptoms and exercise tolerance produced by long-acting bronchodilators, though statistically significant and meeting the minimum clinically important differences in randomized controlled studies, are modest in magnitude and there remains substantial numbers of patients whose dyspnoea persist despite optimal bronchodilator therapy in clinical practice. This is hardly surprising in view of the known pathophysiological processes in COPD detailed above leading to the so-called 'dyspnoea spiral'-chronic dyspnoea leading to physical inactivity and resulting in worsening dyspnoea [23]. In spite of the evidence-based acknowledgement that PR is the most effective modality in improving dyspnoea and exercise tolerance in COPD [24], the fact remains that PR programmes remain universally under-utilized. Nonetheless, with the advancement in mobile health devices and tele-monitoring, accelerated by the present pandemic, home-based PR programmes may prove popular in the near future. The effectiveness of early PR following hospital discharge for COPD exacerbations in reducing mortality has received further support from a recent study [25].

In addition to bronchodilators and supervised exercise training, multiple other interventions have proven effective in reducing dyspnoea in COPD. The use of portable fans blowing on patients' faces [26], inhaled furosemide [27,28], and supplemental oxygen for non-hypoxemic patients during exercise [13] have been shown to reduce dyspnoea and improve exercise tolerance in COPD. Other varieties of modalities shown to reduce dyspnoeic sensation include: inhalation of L-menthol [29], oral opiates [13], breathing cold air and vibration over the intercostal muscles in the parasternal region during inspiration (but not expiration), postural training, and raising of the upper limbs [11]. The myriad factors that affect dyspnoea perception in COPD attest to the multidimensional pathophysiological model elucidated above, just as the various effective therapeutic interventions targeted at reducing NRD further affirm the theory of neuromechanical imbalance in dyspnoea causation.

Current knowledge of dyspnoea in COPD has established the following about the symptom: its clinical importance, pathogenetic factors, effective therapeutic modalities. What is lacking is a precise measurement tool of dyspnoea in COPD that is integrated with patients' actual life situations 
and activity level. Although dyspnoea is a symptom and subjective by nature, an objective means of quantifying the symptom severity is required, in view of the limitations of current patient-reported dyspnoea measurement tools as discussed. The use of diaphragm electromyography (EMGdi), measured using an oesophageal catheter with multiple paired electrodes in determining the ratio of EMGdi during tidal breathing to the maximal volitional value (EMGdi/EMGdi, max), provides a physiologic ratio that is an accurate assessment of the IND [13]. This physiologic quantifiable parameter is closely correlated to level of dyspnoea in healthy individuals and those with COPD [30]. Recent research also evinced that the intimate relationship of IND to dyspnoeic sensation is preserved in COPD patients during incremental exercise testing regardless of the mechanism of dyspnoea relief, i.e., reduction in exertional dyspnoea brought about by bronchodilators, oxygen supplementation and opiates [31]. However, accurate measurement of IND is presently limited to the laboratory by means of invasive testing $[13,32]$. The development of portable and non-invasive recording of EMGdi/EMGdi, max by transcutaneous diaphragmatic EMG, in the form of a wearable device, can potentially provide an objective surrogate measurement of dyspnoea intensity throughout the day, relating it to the patient's situational changes and activities in their daily lives. This can provide a comprehensive analysis of the patient's dyspnoea experience throughout the day, facilitating the implementation of therapeutic interventions that may be customized to the individual patient in his or her usual life circumstances. For instance, the impact of rapid-onset reliever medication to supplement patient's long-acting bronchodilators can be objectively measured. Likewise, patients' dyspnoeic sensation during daily necessities like eating, showering, grooming, sleeping and physical activity can also be recorded for subsequent discussion with the therapist for optimization of measures for dyspnoea control and energy conservation. The concept for such a measurement tool is consistent with the "4 P's" of personalized medicine-predictive, preventive, personalized and participatory [33].

In the meantime, management of the majority of COPD patients according to the GOLD treatment guidelines alluded to at the beginning of this article is based on an initial arbitrary assessment of symptom severity and step-wise addition of medication or non-pharmacological modalities guided by patients' subjective response. For the follow-up of COPD patients in such a general setting, besides subjective symptom evaluation, it may be useful for clinicians to track

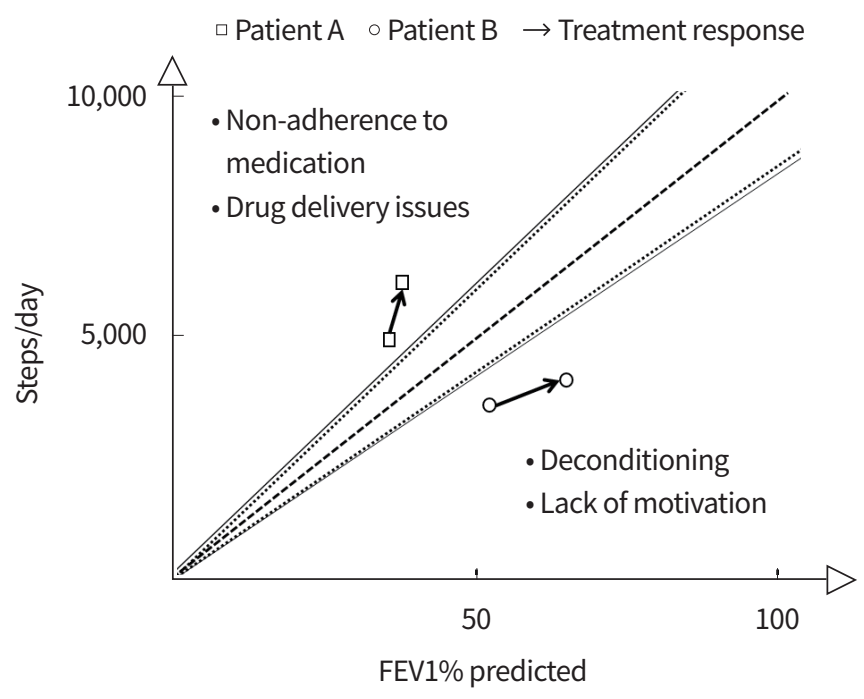

Fig. 1. Possible trajectories of patients' clinical outcomes after initial pharmacotherapy. Arrows represent direction of patients' response to treatment. FEV1\%, forced expiratory volume in 1 second, percent predicted.

two commonly available objective responses to therapy over time-lung function and functional status. These measurements are not only relatively available and repeatable, they also provide longer term prognostic value, especially that of physical activity [34]. Instead of undergoing formal exercise testing, e.g., 6 minute-walk test, patients may be encouraged to simply record their average number of steps taken per day and try to increase this over time. Fig. 1 illustrates that the ideal trajectory of lung function and physical activity improvements should be proportional to each other-as demonstrated by trajectories in the region within the boundaries of the two dashed lines. If a patient does not show adequate lung function improvement after receiving initial bronchodilator therapy (patient $A$ ), the ineffectiveness of prescribed medication may require switching to inhaler devices that emit molecules with finer particle sizes to facilitate drug delivery to smaller airways, after factors such as non-adherence to medication have been excluded. The latter probably requires the use of electronic inhaler monitoring to be made universally available in the near future. Alternatively, if surveillance spirometry shows good improvement with a prescribed inhaler therapy but the trajectory of response is skewed towards increase in lung function without concomitant rise in physical activity level (patient $\mathrm{B}$ ), the patient may require motivation and appropriate exercise training in order to improve their functional status. 


\section{PRECISION AND FUTURE MIEDICINE \\ Kian-Chung Ong}

\section{CONCLUSION}

In summary, dyspnoea is the commonest and most disabling symptom experienced by patients with COPD, and long-term treatment of the disease is aimed primarily at relieving dyspnoea. However, currently available clinical dyspnoea measurement tools lack precision as situational measures in relation to patients' daily activities, and are therefore inadequate for the individualization of patient care. Portable, non-invasive devices providing objective measurements that precisely correlate with dyspnoea experienced by individuals in their daily routine will be invaluable in customizing treatment strategies for COPD patients in future. For the present, improving the outcomes for most COPD patients should be aimed at attaining objective improvements in lung function and functional status, in addition to symptomatic responses with prescribed therapy.

\section{CONFLICTS OF INTEREST}

No potential conflict of interest relevant to this article was reported.

\section{ORCID}

Kian-Chung Ong https://orcid.org/0000-0001-6222-928X

\section{AUTHOR CONTRIBUTIONS}

Conception or design: KCO.

Acquisition, analysis, or interpretation of data: $\mathrm{KCO}$.

Drafting the work or revising: $\mathrm{KCO}$.

Final approval of the manuscript: KCO.

\section{REFERENCES}

1. Global Initiative for Chronic Obstructive Lung Disease. The GOLD Expert Panel: global strategy for the diagnosis, management and prevention of Chronic Obstructive Pulmonary Disease (2021 Report) [Internet]. GOLD; c2021 [cited 2021 May 22]. Available from: https://goldcopd. org/.

2. Svedsater H, Roberts J, Patel C, Macey J, Hilton E, Bradshaw $\mathrm{L}$. Life impact and treatment preferences of individuals with asthma and chronic obstructive pulmonary disease: results from qualitative interviews and focus groups. Adv Ther 2017;34:1466-81.
3. Mullerova H, Lu C, Li H, Tabberer M. Prevalence and burden of breathlessness in patients with chronic obstructive pulmonary disease managed in primary care. PLoS One 2014;9:e85540.

4. Hurst JR, Vestbo J, Anzueto A, Locantore N, Mullerova H, Tal-Singer R, et al. Susceptibility to exacerbation in chronic obstructive pulmonary disease. N Engl J Med 2010;363: 1128-38.

5. Fletcher CM. Standardized questionnaire on respiratory symptoms: a statement prepared and approved by the MRC Committee on the Aetiology of Chronic Bronchitis (MRC breathlessness score). Br Med J 1960;2:1665.

6. Jones PW, Harding G, Berry P, Wiklund I, Chen WH, Kline Leidy N. Development and first validation of the COPD Assessment Test. Eur Respir J 2009;34:648-54.

7. Mahler DA, Weinberg DH, Wells CK, Feinstein AR. The measurement of dyspnea: contents, interobserver agreement, and physiologic correlates of two new clinical indexes. Chest 1984;85:751-8.

8. Antonelli-Incalzi R, Imperiale C, Bellia V, Catalano F, Scichilone N, Pistelli R, et al. Do GOLD stages of COPD severity really correspond to differences in health status? Eur Respir J 2003;22:444-9.

9. Ong KC, Lu SJ, Soh CS. Does the multidimensional grading system (BODE) correspond to differences in health status of patients with COPD? Int J Chron Obstruct Pulmon Dis 2006;1:91-6.

10. Kostikas K, Greulich T, Mackay AJ, Lossi NS, Aalamian-Mattheis M, Nunez X, et al. Treatment response in COPD: does FEV1 say it all?: a post hoc analysis of the CRYSTAL study. ERJ Open Res 2019;5:00243-2018.

11. Jolley CJ, Moxham J. A physiological model of patient-reported breathlessness during daily activities in COPD. Eur Respir Rev 2009;18:66-79.

12. von Leupoldt A, Sommer T, Kegat S, Baumann HJ, Klose $\mathrm{H}$, Dahme B, et al. The unpleasantness of perceived dyspnea is processed in the anterior insula and amygdala. Am J Respir Crit Care Med 2008;177:1026-32.

13. O'Donnell DE, Milne KM, James MD, de Torres JP, Neder JA. Dyspnea in COPD: new mechanistic insights and management implications. Adv Ther 2020;37:41-60.

14. American Thoracic Society. Dyspnea: mechanisms, assessment, and management: a consensus statement. Am J Respir Crit Care Med 1999;159:321-40.

15. O'Donnell DE, Banzett RB, Carrieri-Kohlman V, Casaburi R, Davenport PW, Gandevia SC, et al. Pathophysiology of dyspnea in chronic obstructive pulmonary disease: a 
roundtable. Proc Am Thorac Soc 2007;4:145-68.

16. Carrieri-Kohlman V, Gormley JM, Eiser S, Demir-Deviren S, Nguyen $\mathrm{H}$, Paul SM, et al. Dyspnea and the affective response during exercise training in obstructive pulmonary disease. Nurs Res 2001;50:136-46.

17. Celli B, Blasi F, Gaga M, Singh D, Vogelmeier C, Pegoraro V, et al. Perception of symptoms and quality of life: comparison of patients' and physicians' views in the COPD MIRROR study. Int J Chron Obstruct Pulmon Dis 2017;12:2189-96.

18. Meek PM. Measurement of dyspnea in chronic obstructive pulmonary disease: what is the tool telling you? Chron Respir Dis 2004;1:29-37.

19. Jones P, Lareau S, Mahler DA. Measuring the effects of COPD on the patient. Respir Med 2005;99 Suppl B:S11-8.

20. Calverley PM, Tetzlaff K, Dusser D, Wise RA, Mueller A, Metzdorf $\mathrm{N}$, et al. Determinants of exacerbation risk in patients with COPD in the TIOSPIR study. Int J Chron Obstruct Pulmon Dis 2017;12:3391-405.

21. Nishimura K, Izumi T, Tsukino M, Oga T. Dyspnea is a better predictor of 5-year survival than airway obstruction in patients with COPD. Chest 2002;121:1434-40.

22. Maltais F, Bjermer L, Kerwin EM, Jones PW, Watkins ML, Tombs $L$, et al. Efficacy of umeclidinium/vilanterol versus umeclidinium and salmeterol monotherapies in symptomatic patients with COPD not receiving inhaled corticosteroids: the EMAX randomised trial. Respir Res 2019; 20:238.

23. Decramer M, Janssens W, Miravitlles M. Chronic obstructive pulmonary disease. Lancet 2012;379:1341-51.

24. Singh D, Agusti A, Anzueto A, Barnes PJ, Bourbeau J, Celli $B R$, et al. Global strategy for the diagnosis, management, and prevention of chronic obstructive lung disease: the GOLD science committee report 2019. Eur Respir J 2019; 53:1900164.

25. Lindenauer PK, Stefan MS, Pekow PS, Mazor KM, Priya A, Spitzer KA, et al. Association between initiation of pulmo- nary rehabilitation after hospitalization for COPD and 1-year survival among medicare beneficiaries. JAMA 2020; 323:1813-23.

26. Luckett T, Phillips J, Johnson MJ, Farquhar M, Swan F, Assen T, et al. Contributions of a hand-held fan to self-management of chronic breathlessness. Eur Respir J 2017;50: 1700262.

27. Ong KC, Kor AC, Chong WF, Earnest A, Wang YT. Effects of inhaled furosemide on exertional dyspnea in chronic obstructive pulmonary disease. Am J Respir Crit Care Med 2004;169:1028-33.

28. Jensen D, Amjadi K, Harris-McAllister V, Webb KA, O'Donnell DE. Mechanisms of dyspnoea relief and improved exercise endurance after furosemide inhalation in COPD. Thorax 2008;63:606-13.

29. Kanezaki M, Terada K, Ebihara S. Effect of olfactory stimulation by L-menthol on laboratory-induced dyspnea in COPD. Chest 2020;157:1455-65.

30. Jolley CJ, Luo YM, Steier J, Reilly C, Seymour J, Lunt A, et al. Neural respiratory drive in healthy subjects and in COPD. Eur Respir J 2009;33:289-97.

31. James MD, Masthan MI, Milne KM, Neder JA, O'Donnell $D E$. Exertional dyspnea in COPD: the dominant role of diaphragm activation. In: European Respiratory Society International Congress 2020; 2020 Sep 5-9; Vienna (AT).

32. Domnik NJ, Walsted ES, Langer D. Clinical utility of measuring inspiratory neural drive during Cardiopulmonary Exercise Testing (CPET). Front Med (Lausanne) 2020;7:483.

33. Flores M, Glusman G, Brogaard K, Price ND, Hood L. P4 medicine: how systems medicine will transform the healthcare sector and society. Per Med 2013;10:565-76.

34. Waschki B, Kirsten A, Holz O, Muller KC, Meyer T, Watz H, et al. Physical activity is the strongest predictor of allcause mortality in patients with COPD: a prospective cohort study. Chest 2011;140:331-42. 